\title{
Single-Shot Multi-keV X-Ray Absorption Spectroscopy Using an Ultrashort Laser-Wakefield Accelerator Source
}

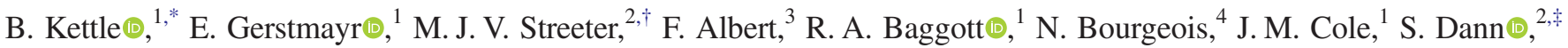 \\ K. Falk, ${ }^{5,6,10}$ I. Gallardo González®,$^{7}$ A. E. Hussein $\odot,{ }^{8}$ N. Lemos, ${ }^{3}$ N. C. Lopes, ${ }^{9}$ O. Lundh, ${ }^{7}$ Y. Ma, ${ }^{2,8}$ S. J. Rose ${ }^{1}{ }^{1}$ \\ C. Spindloe, ${ }^{4}$ D. R. Symes, ${ }^{4}$ M. Šmíd, ${ }^{5}$ A. G. R. Thomas $\odot,{ }^{8,2}$ R. Watt, ${ }^{1}$ and S. P. D. Mangles $\oplus^{1}$ \\ ${ }^{1}$ The John Adams Institute for Accelerator Science, Imperial College London, London, SW7 2AZ, United Kingdom \\ ${ }^{2}$ Physics Department, Lancaster University, Lancaster LA1 4YB, United Kingdom \\ ${ }^{3}$ Lawrence Livermore National Laboratory (LLNL), Livermore, California 94550, USA \\ ${ }^{4}$ Central Laser Facility, STFC Rutherford Appleton Laboratory, Didcot OX11 OQX, United Kingdom \\ ${ }^{5}$ Helmholtz-Zentrum Dresden-Rossendorf, Bautzner Landstrasse 400, 01328 Dresden, Germany \\ ${ }^{6}$ Institute of Physics of the ASCR, Na Slovance 1999/2, 18221 Prague, Czech Republic \\ ${ }^{7}$ Department of Physics, Lund University, P.O. Box 118, S-22100, Lund, Sweden \\ ${ }^{8}$ Center for Ultrafast Optical Science, University of Michigan, Ann Arbor, Michigan 48109-2099, USA \\ ${ }^{9}$ GoLP/Instituto de Plasmas e Fusão Nuclear, Instituto Superior Técnico, U.L., Lisboa 1049-001, Portugal \\ ${ }^{10}$ Technische Universität Dresden, 01062, Dresden, Germany
}

(Received 24 July 2019; revised manuscript received 16 October 2019; published 17 December 2019)

\begin{abstract}
Single-shot absorption measurements have been performed using the multi-keV x rays generated by a laser-wakefield accelerator. A 200 TW laser was used to drive a laser-wakefield accelerator in a mode which produced broadband electron beams with a maximum energy above $1 \mathrm{GeV}$ and a broad divergence of $\approx 15 \mathrm{mrad}$ FWHM. Betatron oscillations of these electrons generated $1.2 \pm 0.2 \times 10^{6}$ photons/eV in the $5 \mathrm{keV}$ region, with a signal-to-noise ratio of approximately $300: 1$. This was sufficient to allow highresolution x-ray absorption near-edge structure measurements at the $K$ edge of a titanium sample in a single shot. We demonstrate that this source is capable of single-shot, simultaneous measurements of both the electron and ion distributions in matter heated to $\mathrm{eV}$ temperatures by comparison with density functional theory simulations. The unique combination of a high-flux, large bandwidth, few femtosecond duration $\mathrm{x}$-ray pulse synchronized to a high-power laser will enable key advances in the study of ultrafast energetic processes such as electron-ion equilibration.
\end{abstract}

DOI: 10.1103/PhysRevLett.123.254801

The extreme conditions present in high-energy-density (HED) matter make it notoriously difficult to study experimentally in the laboratory [1]. X-ray probing is required to investigate the dense interiors of any samples, and any measurements must be made in an ultrashort time frame due to its transient nature and ultrafast dynamics. Because of these difficulties, many HED properties remain uncertain and are an ongoing topic of research. This includes equilibration rates [2], opacities [3,4], equations of state [5], and effects such as continuum lowering [6,7] or nonthermal melting [8]. Understanding these properties is important, for example, for direct and indirect drive fusion experiments $[9,10]$ as well as understanding the internal structure and evolution of large astrophysical objects [11], including that of Earth itself $[12,13]$. X-ray scattering techniques have been very successful in gaining information [14], but provide limited access to the ion temperature and structure without assuming the sample is in thermodynamic equilibrium or knowing the ionization level, Debye temperature, or ion-ion structure factor. The cross sections for $\mathrm{x}$-ray scattering are also quite low, requiring an especially high brightness source such as an $\mathrm{x}$-ray free-electron laser (XFEL) facility.

$\mathrm{X}$-ray absorption measurements can be used to understand both electron and ion dynamics on an atomic scale. For example, the absorption techniques of XANES (x-ray absorption near-edge structure) and EXAFS (extended $\mathrm{X}$-ray absorption fine structure) provide simultaneous measurements of the temperature and structure of the electrons and ions in a sample, as well as the ionization state and more $[15,16]$. The ability to perform $\mathrm{x}$-ray absorption measurements on an ultrafast timescale would enable a significant increase in the fundamental properties that can be derived from experiments involving HED samples, and other ultrafast phenomena. However, for HED experiments, single-shot measurements are crucial, as many of the samples require a large amount of drive energy or complex target designs, making high repetition rates problematic. It is also vital that multi-keV energies are available so that the inner shells of moderate-to-high $Z$ elements can be probed, as well as large sample volumes. Thus, for ultrafast x-ray absorption spectroscopy of HED 

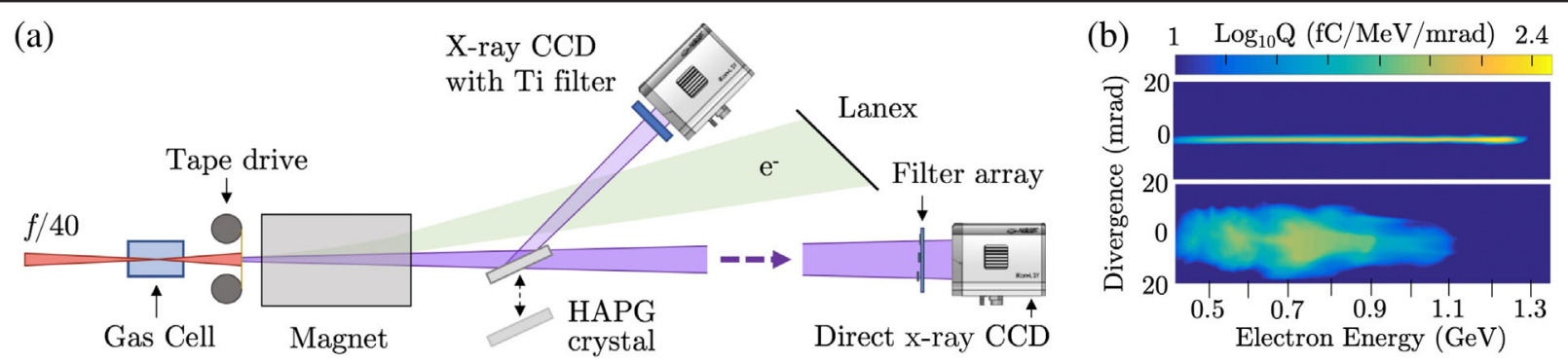

FIG. 1. (a) Experiment setup. The LWFA x rays can be measured on axis or with a high-resolution crystal spectrometer. (b) Electron spectra where, for the first stage of the gas cell, $n_{e}=1.2 \times 10^{18} \mathrm{~cm}^{-3}$ (top) and $n_{e}=2.6 \times 10^{18} \mathrm{~cm}^{-3}$ (bottom).

samples to produce valuable and reliable data, a highbrightness smooth broadband spectrum $x$-ray probe in the multi-keV region is required. The National Ignition Facility and the Omega Laser Facility have both been developing EXAFS diagnostics in the multi-keV regime [17,18]. These sources, however, require hundreds of joules of backlighter energy, and are over 100 ps in duration. In general, synchrotron facilities lack the required ultrashort pulse duration, and laser-plasma-based approaches suffer additionally from low brightness and relatively noisy spectra [19]. XFELs have the required flux and pulse duration, but are monochromatic in nature. Increased bandwidth techniques are being investigated [20]; however they lack a smooth broadband spectrum, making absorption measurements difficult.

A viable solution is to perform $\mathrm{x}$-ray absorption measurements using a laser-wakefield accelerator (LWFA). These are the only currently available sources that provide bright bursts of broadband $\mathrm{x}$ rays on the femtosecond timescale [21] and their application in HED science has become an active research field. To date, however, the source flux has required absorption spectra to be integrated over many shots or the photon energy range has been limited to lower energies ( $\mathrm{keV}$ or less) [22-24]. In this Letter we present the first single-shot multi-keV XANES measurement using the ultrashort $\mathrm{x}$ rays from a LWFA source. This was achieved by operating the LWFA in a tailored mode to generate high $\mathrm{x}$-ray flux (more than 100 times that of previous measurements) and multi-keV photon energies, in tandem with an efficient and highresolution single optic detector.

The experiment was conducted using the Gemini Laser at the Central Laser Facility (UK). An overview of the experiment setup can be seen in Fig. 1(a) [25]. The drive laser $(800 \mathrm{~nm})$ was focused using an $f / 40$ geometry into a gas cell. Each laser pulse (provided at $0.05 \mathrm{~Hz}$ ) had a duration of $47 \pm 5 \mathrm{fs}$ and contained $9 \pm 0.3 \mathrm{~J}$. These pulses were focused to a spot of $(50 \pm 2) \times(43 \pm 1) \mu \mathrm{m}^{2}$ FWHM, with the central FWHM containing $43 \pm 2 \%$ of the energy. This provided an on-target intensity of $(4.9 \pm 0.6) \times 10^{18} \mathrm{~W} / \mathrm{cm}^{2}$ and an average laser strength parameter of $a_{0} \approx 1.5$. As the laser pulse traveled through the gas, it drove a LWFA [26], where the electrons liberated from the atoms were expelled by the ponderomotive force of the laser, creating an ion cavity in its wake. The strong electric field inside the cavity can subsequently accelerate electrons to $\mathrm{GeV}$ energies in just a few centimeters $[27,28]$. Our LWFA operated using a two-stage gas cell $[29,30]$. The first stage ( $3 \mathrm{~mm}$ long) was filled with a $98 \% \mathrm{He}+2 \% \mathrm{~N}_{2}$ gas mix, and the second stage (19.6 mm long) was filled with He. Electrons were injected in the first stage using ionization injection [31,32]. The second stage provided the acceleration of the electrons. While in the back of the ion cavity the electrons perform betatron oscillations around the laser axis, producing high-energy $\mathrm{x}$ rays $[33,34]$. The on-axis intensity spectrum is synchrotronlike and characterized by the critical energy $E_{\text {crit }}$ and is given by $d^{2} I /(d E d \Omega) \propto\left(E / E_{\text {crit }}\right)^{2} \mathcal{K}_{2 / 3}^{2}\left[E /\left(2 E_{\text {crit }}\right)\right]$, where $\mathcal{K}_{2 / 3}[x]$ is a modified Bessel function of order 2/3. The x-ray pulse emission is of similar duration to that of the electron bunch, which is typically on the order of $10 \mathrm{fs}$ [35]. The source size is on the order of microns and the emission is directed in a tight cone along the propagation axis, with a divergence of $\lesssim 20 \mathrm{mrad}$ FWHM.

After the $\mathrm{x}$ rays exit the accelerator, a replenishable tape drive was used to dump the remaining laser energy, and a high strength magnet $(\approx 0.8 \mathrm{~T}, 10 \mathrm{~cm})$ was used as an electron energy spectrometer. The tape is made of polyimide plastic and is $25 \mu \mathrm{m}$ thick. It has a transmission of over $90 \%$ for $\mathrm{x}$-ray energies over $5 \mathrm{keV}$. Two example electron spectra can be seen in Fig. 1(b). The x-ray spectrum was measured with high resolution using the reflection from a crystal (protected from laser damage by the sacrificial tape drive) or directly imaged through a set of metallic filters to estimate the broadband spectrum [34]. The high-resolution spectral measurements of the $\mathrm{x}$ rays were made over a range of $\approx 80 \mathrm{eV}$. A $100 \mu$ m thick HAPG (highly annealed pyrolytic graphite) crystal with $\approx 0.1^{\circ}$ mosaic spread on a $2 \times 6 \mathrm{~cm}$ BK7 substrate was used. Mosaic crystals (as opposed to perfect crystals) are made up of many smaller crystallite planes that have a random nature to their orientation. The angles of these planes are seen to have a normal distribution with a width of less than a degree. However, this spread in crystallite angles throughout the crystal structure is responsible for increasing the reflection efficiency, as the Bragg condition for any 

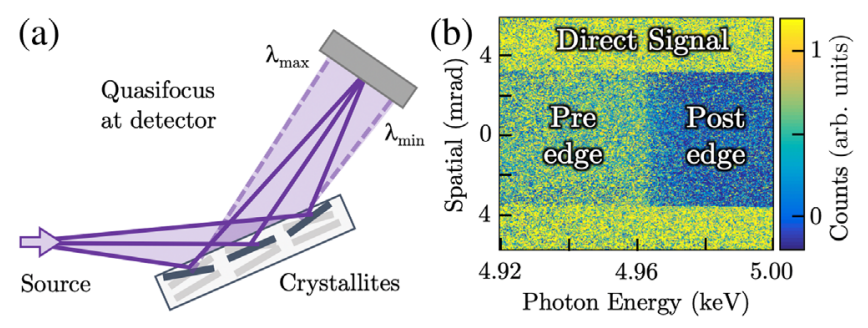

FIG. 2. (a) Illustration of mosaic quasifocusing. Solid rays depict a single wavelength focused at the detector. Dashed rays indicate the minimum or maximum wavelength (dictated by the source divergence). (b) Single-shot crystal spectrometer image corrected for the crystal reflectivity profile.

given photon wavelength can now be satisfied over a larger surface area of the crystal. This effect is known as quasifocusing and provides reflection efficiencies of over 10 times that of a perfect crystal, while maintaining the high-spectral resolution if used in a 1-to-1 geometry [36]. See Fig. 2(a) for an illustration of this operation. The source to crystal (and crystal to detector) distance was $41 \pm 1 \mathrm{~cm}$. Ray-tracing simulations estimate the instrument function of this spectrometer to have a width of $\approx 2.2 \mathrm{eV}$, consistent with the estimates of Zastrau et al. [37]. This resolution is dominated by quasifocusing of the mosaic crystal, as opposed to the single crystal plane broadening or source broadening effects (as the LWFA source is on the order of microns). By using a single high-reflectivity optic we have optimized the overall efficiency of the x-ray detector while maintaining high-spectral resolution. A $10 \mu \mathrm{m}$ thick titanium sample strip was placed in front of the CCD to record absorption features around its $K$ edge $(4966 \mathrm{eV})$.

For plasma densities of $n_{e}=1.2 \times 10^{18} \mathrm{~cm}^{-3}$ and $n_{e}=$ $2.3 \times 10^{18} \mathrm{~cm}^{-3}$ in the first and second cell stages, respectively, electron beams with a maximum energy at $1.2 \mathrm{GeV}$ and a divergence of $1 \mathrm{mrad}$ were observed on the magnetic spectrometer [see Fig. 1(b), top]. However, we found that we were able to generate 10 times more $\mathrm{x}$-ray flux by increasing the plasma density to $2.6 \times 10^{18} \mathrm{~cm}^{-3}$ in both stages. At this density, the observed electron beam had a lower maximum energy, but a greater total charge and transverse momentum [see Fig. 1(b), bottom]. This also increased the $\mathrm{x}$-ray divergence to $\approx 15 \mathrm{mrad}$ FWHM. As the divergence of the $\mathrm{x}$-ray source provides the range of different incident angles upon the crystal spectrometer and the spectral spread of the detector is achieved by satisfying the Bragg condition at different angles, a more divergent beam leads to a wider accessible spectral range. For the high-flux shots the direct filter pack measured a mean critical energy of $E_{\text {crit }}=9.9 \pm 1.5 \mathrm{keV}$, and the entire beam contained $(7.2 \pm 2.8) \times 10^{5}$ photons $/ \mathrm{eV}$ at $5 \mathrm{keV}$, comparable to the highest $\mathrm{x}$-ray flux observed in previous LWFA measurements $[30,38]$. The shot-to-shot standard deviation here is combined with the systematic errors in quadrature.
A single-shot image from the crystal spectrometer can be seen in Fig. 2(b). It has been background corrected, and the spatial variations in the signal due to the mosaic crystal structure have been folded out (see Supplemental Material for further details [39]). The horizontal axis corresponds to the $\mathrm{x}$-ray energy, while the vertical axis provides spatial information perpendicular to the dispersion direction. The shadow of the titanium sample foil along the central region provides the absorption profile around the inner $K$ shell, whereas the direct signal either side measures the $x$-ray yield and smoothness.

In the direct signal region for the brightest shot, we measure $(1.2 \pm 0.2) \times 10^{6}$ photons/eV. Assuming a Poisson distribution, the random statistical noise should be $\sqrt{N_{\mathrm{ph}}}$, where $N_{\mathrm{ph}}$ is the number of photons; i.e., we should have a signal-to-noise level of $\approx 1100: 1$ per eV. Our direct signal exhibits a signal-to-noise ratio of 300:1 (standard deviation in the photon yield per $\mathrm{eV}, 0.34 \%$ of the signal level). One of the main contributions to the noise comes from an underlying background that is combined with the $\mathrm{x}$-ray signal from the crystal reflection. This noise is present even on shots where the $\mathrm{x}$-ray crystal (but not the CCD) was removed from the beam line, indicating that the source is not inherent to the measurement. The background is seen to scale linearly with the total charge of the electron beam. Single-hit photon analysis of low-charge shots also suggest that the CCD hits are from a broad spectra of hard $\mathrm{x}$ rays and occasional high-energy particle hits. This is consistent with the accelerated electrons interacting with the target chamber and creating secondary particles which produce the background noise. The measured standard deviation noise on an x-ray shot (with the crystal in place) is found to be on average $\lesssim 12 \%$ higher than that of a background shot (without the crystal). Assuming the noise sources add in quadrature, this suggests the statistical noise inherent in the betatron signal is less than half of the electron beam produced background noise. $\sigma_{\text {signal }}=$ $\sigma_{\mathrm{bg}} \sqrt{\left(\sigma_{\mathrm{all}} / \sigma_{\mathrm{bg}}\right)^{2}-1}$. Importantly, it should therefore be possible to significantly reduce the background with improved shielding and appropriate electron beam dumping.

Figure 3 depicts the measured absorption profile for a single shot (solid black curve). It is compared alongside reference data for titanium taken previously at a synchrotron facility [40] (dotted red curve). To facilitate the comparison we have used a standard XANES procedure for normalizing the profile [41]. This has the added benefit of not requiring a direct spectrum (no sample absorption) to be measured, as long as the signal is relatively smooth and stable (a key strength of the betatron radiation from the LWFA). The reference data, which already have an inherent instrument width, have had a $2 \mathrm{eV}$ FWHM instrument function applied (to match our detector resolution).

The single-shot measurement provides a clear match to the rising slope structure $(<4970 \mathrm{eV})$ as well as emulating 


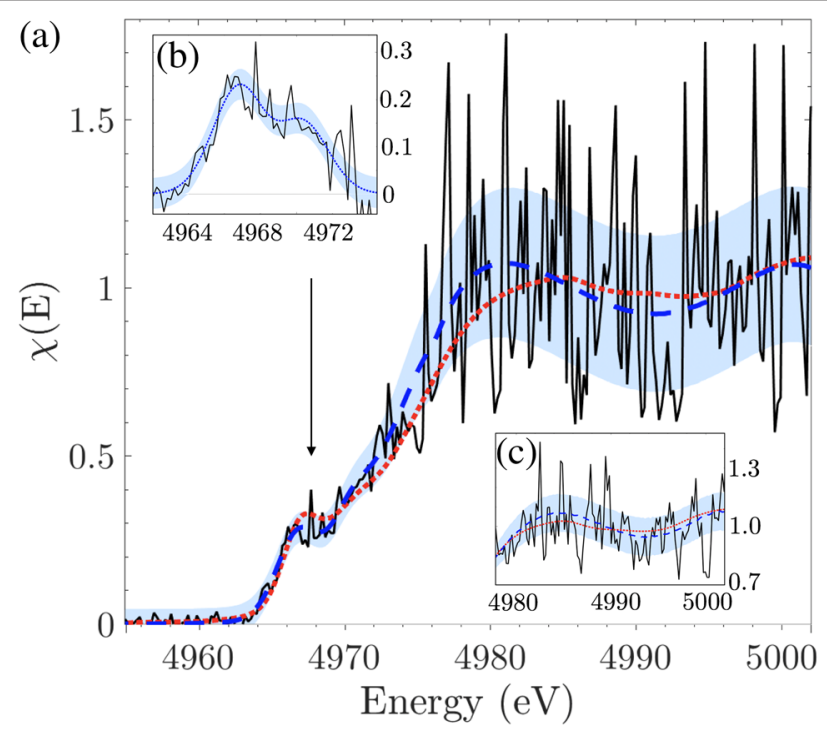

FIG. 3. (a) Single-shot normalized absorption data (solid black curve) compared to a synchrotron reference measurement [40] (dotted red curve). The fitted profile for our data is given (dashed blue curve) with the light blue area indicating the measurement error. (b) Double Gaussian fit to the preedge features. (c) The same result as in (a), but averaged over 11 shots.

the preedge feature at $4967 \mathrm{eV}$. This preedge feature is a set of forbidden transitions into the $3 d$ shell, allowed by $3 d-4 p$ mixing, and provides information about the bonding properties of the sample [16]. The underlying slope of the edge corresponds to the density of free states, and provides the temperature of the electron distribution (which was $300 \mathrm{~K}$ in our case).

To examine the profile we fit various structures to the different aspects of its shape. First we fit a sigmoid function to replicate the Fermi distribution of the electrons, where the width is proportional to the temperature. We fit a double Gaussian to the two preedge forbidden transitions, and a fifth-order polynomial to the oscillatory component of the XANES interference features after the edge. Figure 3(b) illustrates the double Gaussian fit after the sigmoid subtraction. These fit components are combined and an error bar equal to the standard error of the fit combined with the error in the crystal reflectivity is added; see Fig. 3, dashed blue line and gray shaded areas, respectively. This fitting procedure allows us to quantify how well our data agree with the reference data.

We assess our resolution on a single shot, by studying the fitting fluctuations over several consecutive shots. We observe a fit to the edge position with a standard error of $0.17 \mathrm{eV}$. The standard deviation in the position of the foot (10\% value) before the preedge features is $0.28 \mathrm{eV}$. Assuming a Fermi distribution of the electrons we estimate this would allow a resolvable change in electron temperature of $\approx 0.4 \mathrm{eV}$ on a single shot. The amplitude of the preedge Gaussians has an $18 \%$ error. In summary, on a single-shot measurement we are capable of quantitatively resolving electronic structure information and electron temperature with sub-eV accuracy.

The postedge modulations in the profile also contain valuable information regarding the ion component of the sample. It has been estimated that a signal-to-noise ratio of 1000: 1 is required to make a high-quality EXAFS measurement of the ion peak beyond the edge, with good statistics [42]. We can emulate the expected improvements to the signal-to-noise ratio that will be achieved with improved electron beam shielding by averaging the data over 11 shots. The inset of Fig. 3 depicts the measured absorption profile (solid black curve), our resulting fit (blue dashed curve) with shaded error bars, and the synchrotron reference (red dotted curve) [40]. The error magnitude in the signal region postedge has been reduced by a factor of 2. From the noise discussion before, it was seen that the background noise present in our data contributes at least twice that of the $\mathrm{x}$-ray signal from the crystal reflection. Therefore, a signal-to-noise ratio similar to the integrated shots should be achieved (or bettered) for a single shot with an improved electron beam dump and detector shielding. The contrast in the absorption profile can also be improved by a factor of 2 by choosing an optimal sample thickness (1/e absorption depth). From comparison to a range of density functional theory (DFT) simulations at different ion temperatures, the resolution achieved in the postedge modulation structure (assuming noise reduction) should be sufficient to see a change of $\approx 0.5 \mathrm{eV}$ in ion temperature (via the "flattening" of the modulation structure). This is extremely valuable information, especially in tandem with the electron temperature accessed via the absorption edge slope.

With that in mind, we discuss the possibility of investigating a nonequilibrated HED sample. Figure 4 depicts DFT results for titanium using python based projectoraugmented wave method (GPAW) [43]. A $3 \mathrm{eV}$ instrument function is applied to (conservatively) emulate experimental measurements. The normalized absorption profile is

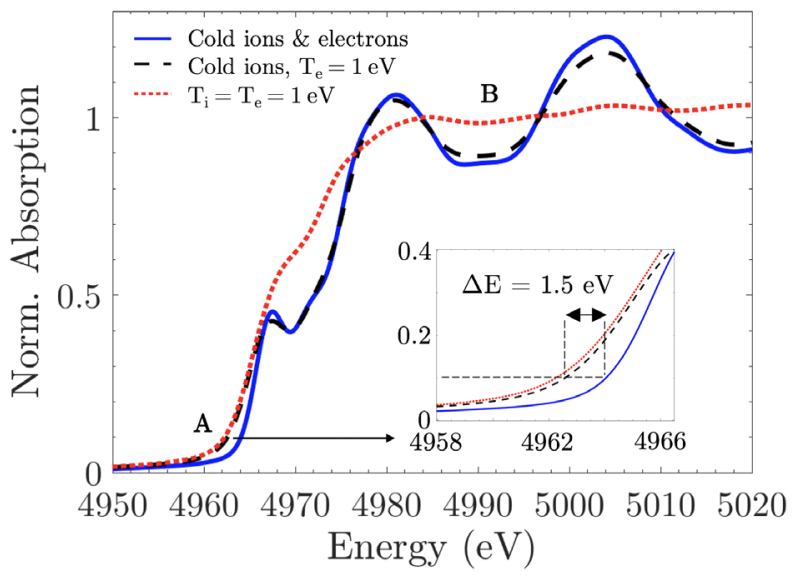

FIG. 4. DFT simulations for titanium at various heating conditions (room temperature, nonequilibrated, and $T=1 \mathrm{eV}$ ). 
given for three scenarios: a cold foil (solid blue curve), a foil in a nonequilibrated situation with $T_{e}=1 \mathrm{eV}$ and cold ions (dashed black curve), and, finally, a foil with $T_{e}=$ $T_{i}=1 \mathrm{eV}$ (dotted red curve). The effect on the preedge profile due to the increase in electron temperature can be clearly seen in the latter two cases with a $1.5 \mathrm{eV}$ shift in the position of the foot. This is highlighted in Fig. 4 with the $A$ marker and the enlarged inset. Our single-shot data are more than capable of resolving such a change in the profile. The loss of the postedge modulations is only evident in the final case (highlighted by $B$ ), when the ion temperature is increased and the radial distribution function flattens. For any single measured absorption profile it would be possible to independently deduce the electronic and ionic structure and temperature using these independent shifts in the absorption structure. This would, for example, allow the electron-ion equilibration rate to be directly diagnosed, a highly sought-after measurement [2].

With more high-intensity lasers coming on-line around the world that can generate the required LWFA x-ray source, especially in tandem with sample drivers such as XFELs or high-energy laser systems, single-shot x-ray absorption spectroscopy offers great capabilities in making ultrafast measurements of many fundamental processes in HED science. This includes the use of XANES for electronion equilibration rate measurements as described above, but also high-quality ultrafast $\mathrm{x}$-ray absorption data could be used to investigate continuum lowering effects [6] or informing solar opacity debates [3]. Commercial and industrial applications of multi-keV absorption also become feasible. For example, the ultrafast phase changes that can be measured via the EXAFS structures are important in battery [44] and memory storage [45] studies. Single-shot measurements would increase the efficiency of such studies and the femtosecond pulse length would resolve the phase dynamics further. Finally, development of the LWFA x-ray properties is an ongoing and active research area. Any advances will extend the capabilities of this technique. For example, an increased $\mathrm{x}$-ray flux will allow higher spectral resolution measurements by allowing less efficient perfect crystals to be used in the x-ray spectrometer.

In conclusion, we have demonstrated a novel experimental technique for performing single-shot ultrafast $\mathrm{x}$-ray absorption measurements of the electronic structure of a sample. These measurements were made possible using the smooth broadband multi-keV synchrotron radiation produced by a laser-driven plasma wakefield accelerator. To achieve a single-shot measurement we generated a highenergy and high-charge electron beam from the accelerator to produce an $\mathrm{x}$-ray flux of $>10^{6}$ photons/eV and implemented a suitable high-resolution and high-efficiency x-ray spectrometer using a single reflection optic. We were able to perform XANES measurements of room temperature titanium, with the results in agreement with measurements taken at a third generation light source. Our data provide not only the electron temperature distribution but information on any additionally supported preedge transitions. With minor improvements to our experimental setup one should be able to access the ion component further from the absorption edge and make ultrafast single-shot EXAFS measurements of mid-to-high $Z$ elements. This will allow the simultaneous measurement of the electronic and ionic temperature and structure of high-energy-density samples on a timescale of tens of femtoseconds, making significant new areas of research possible.

We wish to acknowledge the support of the staff at the Central Laser Facility. This project has received funding from the European Research Council (ERC) under the European Union's Horizon 2020 research and innovation programme (Grant Agreement No. 682399), as well as the U.S. Department of Energy Office for Fusion Energy Sciences, Project No. DE-SC0019186 and the Knut and Alice Wallenberg Foundation.

*b.kettle@imperial.ac.uk

'Present address: The John Adams Institute for Accelerator Science, Imperial College London, London, SW7 2AZ, United Kingdom.

${ }^{\star}$ Present address: Central Laser Facility, STFC Rutherford Appleton Laboratory, Didcot OX11 0QX, United Kingdom.

${ }^{\S}$ Present address: Center for Ultrafast Optical Science, University of Michigan, Ann Arbor, Michigan 481092099, USA.

[1] R. P. Drake, High-Energy-Density Physics, 2nd ed. (Springer International Publishing, Cham, 2018).

[2] B. I. Cho, K. Engelhorn, A. A. Correa, T. Ogitsu, C. P. Weber, H. J. Lee, J. Feng, P. A. Ni, Y. Ping, A. J. Nelson et al., Phys. Rev. Lett. 106, 167601 (2011).

[3] J. E. Bailey, T. Nagayama, G. P. Loisel, G. A. Rochau, C. Blancard, J. Colgan, P. Cosse, G. Faussurier, C. J. Fontes, F. Gilleron et al., Nature (London) 517, 56 (2015).

[4] B. Kettle, T. Dzelzainis, S. White, L. Li, B. Dromey, M. Zepf, C. L. S. Lewis, G. Williams, S. Künzel, M. Fajardo et al., Phys. Rev. E 94, 023203 (2016).

[5] P. Renaudin, C. Blancard, J. Clérouin, G. Faussurier, P. Noiret, and V. Recoules, Phys. Rev. Lett. 91, 075002 (2003).

[6] D. J. Hoarty, P. Allan, S. F. James, C. R. D. Brown, L. M. R. Hobbs, M. P. Hill, J. W. O. Harris, J. Morton, M. G. Brookes, R. Shepherd et al., Phys. Rev. Lett. 110, 265003 (2013).

[7] O. Ciricosta, S. M. Vinko, B. Barbrel, D. S. Rackstraw, T. R. Preston, T. Burian, J. Chalupský, B. I. Cho, H. K. Chung, G. L. Dakovski et al., Nat. Commun. 7, 11713 (2016).

[8] N. Hartley, J. Grenzer, W. Lu, L. Huang, Y. Inubushi, N. Kamimura, K. Katagiri, R. Kodama, A. Kon, V. Lipp et al., High Energy Density Phys. 32, 63 (2019).

[9] R. S. Craxton, K. S. Anderson, T. R. Boehly, V. N. Goncharov, D. R. Harding, J. P. Knauer, R. L. McCrory, P. W. McKenty, D. D. Meyerhofer, J. F. Myatt et al., Phys. Plasmas 22, 110501 (2015).

[10] J. Lindl, Phys. Plasmas 2, 3933 (1995).

[11] B. A. Remington, Plasma Phys. Controlled Fusion 47, A191 (2005). 
[12] J. H. Nguyen and N. C. Holmes, Nature (London) 427, 339 (2004).

[13] S. White, G. Nersisyan, B. Kettle, T. Dzelzainis, K. McKeever, C. Lewis, A. Otten, K. Siegenthaler, D. Kraus, M. Roth et al., High Energy Density Phys. 9, 573 (2013).

[14] S. H. Glenzer and R. Redmer, Rev. Mod. Phys. 81, 1625 (2009).

[15] J. J. Rehr and R. C. Albers, Rev. Mod. Phys. 72, 621 (2000).

[16] F. Dorchies and V. Recoules, Phys. Rep. 657, 1 (2016).

[17] A. Krygier, F. Coppari, G. E. Kemp, D. B. Thorn, R. S. Craxton, J. H. Eggert, E. M. Garcia, J. M. McNaney, H.-S. Park, Y. Ping, B. A. Remington, and M. B. Schneider, Rev. Sci. Instrum. 89, 10F114 (2018).

[18] Y. Ping, F. Coppari, D. G. Hicks, B. Yaakobi, D. E. Fratanduono, S. Hamel, J. H. Eggert, J. R. Rygg, R. F. Smith, D. C. Swift et al., Phys. Rev. Lett. 111, 065501 (2013).

[19] A. Mančić, A. Lévy, M. Harmand, M. Nakatsutsumi, P. Antici, P. Audebert, P. Combis, S. Fourmaux, S. Mazevet, O. Peyrusse et al., Phys. Rev. Lett. 104, 035002 (2010).

[20] E. Prat, M. Calvi, and S. Reiche, J. Synchrotron Radiat. 23, 874 (2016).

[21] K. Ta Phuoc, R. Fitour, A. Tafzi, T. Garl, N. Artemiev, R. Shah, F. Albert, D. Boschetto, A. Rousse et al., Phys. Plasmas 14, 080701 (2007).

[22] M.Z. Mo, Z. Chen, S. Fourmaux, A. Saraf, S. Kerr, K. Otani, R. Masoud, J.-C. Kieffer, Y. Tsui, A. Ng et al., Phys. Rev. E 95, 053208 (2017).

[23] M. Šmíd, I. G. González, H. Ekerfelt, J. B. Svensson, M. Hansson, J. C. Wood, A. Persson, S. P. D. Mangles, O. Lundh, and K. Falk, Rev. Sci. Instrum. 88, 063102 (2017).

[24] B. Mahieu, N. Jourdain, K. T. Phuoc, F. Dorchies, J.-P. Goddet, A. Lifschitz, P. Renaudin, and L. Lecherbourg, Nat. Commun. 9, 3276 (2018).

[25] See http://dx.doi.org/10.5281/zenodo.3340697, dataset available online.

[26] T. Tajima and J. M. Dawson, Phys. Rev. Lett. 43, 267 (1979).

[27] W. P. Leemans, B. Nagler, A. J. Gonsalves, C. Toth, K. Nakamura, C. G. R. Geddes, E. S. C. B. Esarey, C. B. Schroeder, and S. M. Hooker, Nat. Phys. 2, 696 (2006).

[28] S. Kneip, S. R. Nagel, S. F. Martins, S. P. D. Mangles, C. Bellei, O. Chekhlov, R. J. Clarke, N. Delerue, E. J. Divall, G. Doucas et al., Phys. Rev. Lett. 103, 035002 (2009).

[29] B. B. Pollock, C. E. Clayton, J.E. Ralph, F. Albert, A. Davidson, L. Divol, C. Filip, S. H. Glenzer, K. Herpoldt, W. Lu et al., Phys. Rev. Lett. 107, 045001 (2011).
[30] A. E. Hussein, N. Senabulya, Y. Ma, M. J. V. Streeter, B. Kettle, S. J. D. Dann, F. Albert, N. Bourgeois, S. Cipiccia, J. M. Cole et al., Sci. Rep. 9, 3249 (2019).

[31] A. Pak, K. A. Marsh, S. F. Martins, W. Lu, W. B. Mori, and C. Joshi, Phys. Rev. Lett. 104, 025003 (2010).

[32] C. McGuffey, A. G. R. Thomas, W. Schumaker, T. Matsuoka, V. Chvykov, F. J. Dollar, G. Kalintchenko, V. Yanovsky, A. Maksimchuk, K. Krushelnick et al., Phys. Rev. Lett. 104, 025004 (2010).

[33] A. Rousse, K. T. Phuoc, R. Shah, A. Pukhov, E. Lefebvre, V. Malka, S. Kiselev, F. Burgy, J.-P. Rousseau, D. Umstadter et al., Phys. Rev. Lett. 93, 135005 (2004).

[34] S. Kneip, C. McGuffey, J. L. Martins, S. F. Martins, C. Bellei, V. Chvykov, F. Dollar, R. Fonseca, C. Huntington, G. Kalintchenko et al., Nat. Phys. 6, 980 (2010).

[35] O. Lundh, J. Lim, C. Rechatin, L. Ammoura, A. Ben-Ismaïl, X. Davoine, G. Gallot, J.-P. Goddet, E. Lefebvre, V. Malka, and J. Faure, Nat. Phys. 7, 219 (2011).

[36] H. Legall, H. Stiel, M. Schnürer, M. Pagels, B. Kanngießer, M. Müller, B. Beckhoff, I. Grigorieva, A. Antonov, V. Arkadieve, and A. Bjeoumikhov, J. Appl. Crystallogr. 42, 572 (2009).

[37] U. Zastrau, A. Woldegeorgis, E. Förster, R. Loetzsch, H. Marschner, and I. Uschmann, J. Instrum. 8, P10006 (2013).

[38] J. M. Cole, J. C. Wood, N. C. Lopes, K. Poder, R. L. Abel, S. Alatabi, J. S. J. Bryant, A. Jin, S. Kneip, K. Mecseki et al., Sci. Rep. 5, 13244 (2015).

[39] See Supplemental Material at http://link.aps.org/ supplemental/10.1103/PhysRevLett.123.254801 for technical details related to the data processing of the x-ray diagnostics in the main text.

[40] P. Bleith, M. Valla, P. Nováka, and C. Villevieille, J. Mater. Chem. A 2, 12513 (2014).

[41] M. Newville, Rev. Mineral. Geochem. 78, 33 (2014).

[42] F. Albert, A. G. R. Thomas, S. P. D. Mangles, S. Banerjee, S. Corde, A. Flacco, M. Litos, D. Neely, J. Vieira, and Z. Najmudin, Plasma Phys. Controlled Fusion 56, 084015 (2014).

[43] J. Enkovaara, C. Rostgaard, J. J. Mortensen, J. Chen, M. Dułak, L. Ferrighi, J. Gavnholt, C. Glinsvad, V. Haikola, H. A. Hansen et al., J. Phys. Condens. Matter 22, 253202 (2010).

[44] J. Wang, Y. K. Chen-Wiegart, C. Eng, Q. Shen, and J. Wang, Nat. Commun. 7, 12372 (2016).

[45] P. Zalden, F. Quirin, M. Schumacher, J. Siegel, S. Wei, A. Koc, M. Nicoul, M. Trigo, P. Andreasson, H. Enquist et al., Science 364, 1062 (2019). 